\title{
Presenting multiple auditory signals using multiple sound cards in Visual Basic 6.0
}

\author{
JASON S. CHAN and CHARLES SPENCE \\ University of Oxford, Oxford, England
}

\begin{abstract}
In auditory research, it is often desirable to present more than two auditory stimuli at any one time. Although the technology has been available for some time, the majority of researchers have not utilized it. This article provides a simple means of presenting multiple, concurrent, independent auditory events, using two or more different sound cards installed within a single computer. By enabling the presentation of more auditory events, we can hope to gain a better understanding of the cognitive and attentional processes operating under more complex and realistic scenes, such as that embodied by the cocktail party effect. The software requirements are Windows 98SR2/Me/NT4/2000/XP, Visual Basic 6.0, and DirectX 7.0 or above. The hardware requirements are a Pentium II, $128 \mathrm{MB}$ RAM, and two or more different sound cards.
\end{abstract}

Although there have been extensive developments in the presentation of visual stimuli in recent years, there have been far fewer developments in the presentation of auditory stimuli. In particular, one critical limitation in auditory research has been the difficulty associated with presenting multiple auditory stimuli, such as multiple voices, at the same time from different spatial locations, as when trying to study the cocktail party problem. To date, research concerning this phenomenon has utilized, at most, two auditory signals (Cherry, 1953, 1954; Nelson, Bolia, Ericson, \& McKinley, 1998; Spieth, Curtis, \& Webster, 1954; Wood \& Cowan, 1995a, 1995b; Yost, 1997). To the best of our knowledge, none of the issues addressed in these experiments has been readdressed with more than two voices, with the exception of the studies reported by Yost.

Lavie and colleagues (Lavie \& Fox, 2000; Lavie \& Tsal, 1994) found that in the visual system, as the perceptual load of a task increased, participants increasingly selectively attended only to relevant target stimuli and ignored irrelevant distractor stimuli. This could also be the case in the auditory modality. However, it is difficult to test this hypothesis without increasing the perceptual load in the auditory modality. The aim of the present article is to demonstrate how multiple auditory events can be presented simultaneously, using Visual Basic 6.0 (Microsoft Corp., Redmond, WA), in the way we have been using it to study the auditory cocktail party problem (e.g., Chan \& Spence, 2002). ${ }^{1}$ This question can be better studied by presenting more than two separate concurrent auditory events at once to increase the perceptual

Correspondence concerning this article should be addressed to J. Chan, Department of Experimental Psychology, University of Oxford, South Parks Road, Oxford OX1 3UD, England (e-mail: jason. chan@psy.ox.ac.uk). load in the auditory system. The method presented here provides one example of how auditory stimuli from several sound files can be presented simultaneously. The majority of sound cards have two audio channels (i.e., a right and a left channel). It is possible to install two or more such sound cards into a single computer and, by so doing, present four or more concurrent independent auditory events.

Historically, Windows programming has not been capable of supporting a greater number of channels, sample rates, and sample formats with low latency (Steinberg, 2002). However, with the more current Windows device media (WDM) and Mac programming and faster computing power, this is no longer the case. Although sound cards with more than two channels have been available for several years, it was only about 2 years ago (i.e., in 2000) that the sound card industry developed a universal standard to program these sound cards. This article does not address this method for stimulus presentation. However, it will demonstrate how to play multiple sound files simultaneously, using multiple sound cards instead.

There are also other third-party developments that are able to present more than two independent auditory stimuli. OpenAL is an open audio library and is designed to integrate seamlessly with OpenGL (OpenAL, 2000). ASIO is optimized for multiple channels, high sampling rates, and sample formats. The main advantage of ASIO over DirectX lies in its ability to do all of those things with low latency (i.e., live streaming playback and recording). DirectSound can provide latencies as low as $20 \mathrm{msec}$ (Microsoft, 2000). However, if DirectSound must emulate hardware features in software, latencies can be as long as 100-150 msec (Microsoft, 2000). ASIO is capable of sound latencies of approximately 3-11 msec (Mars, 2002). This is particularly useful when one is trying to stream audio from different audio sources that require a sound 
monitor. It is unnecessary to program with these add-ons if the onsets of the sound stimuli are not time sensitive. This article does not necessarily give an example of the best method for delivering multiple sounds, but merely one of several possible options now available for delivering multiple sounds simultaneously.

The constant updates of Microsoft's DirectX makes third-party software virtually unnecessary. DirectX is an advanced programming interface that is compatible with most programming languages. It allows the programmer to control directly the hardware of each component though software implementation. For the purposes of this paper, DirectX accesses special hardware features of the sound cards (e.g., panning and volume control), without the need for additional software. Before the advent of DirectSound (a subfunction of DirectX), programmers were required to incorporate the Windows Media Player into their program. This only allowed for basic functions (e.g., play, stop, and pause).

The software required consists of Windows 98SR2/ Me/NT4/2000/XP (Microsoft Corp., Redmond, WA), Visual Basic 6.0, and the DirectX 7.0 (Microsoft Corp., Redmond, WA) or above plug-in. The hardware requirements are two or more different sound cards. These sound cards should not be of the same model but can be produced by the same manufacturer. This is because, although Windows will detect each sound card, the software driver operating each sound card will not, when two identical sound cards are installed in parallel. The software driver will operate only one sound card at any given time. The minimum CPU should be a Pentium II. One or more free motherboard slots are required, depending on whether or not a sound card is already installed in the computer. The amount of RAM is dependent on the number and size of the sound files that one intends to buffer. For example, loading three 2-min speech audio files will require a minimum of $128 \mathrm{MB}$ of RAM for the speech to play smoothly. If speech is broken and erratic, it could be an indication that more RAM is required.

\section{Method}

It is best to install each sound card individually. Insert one sound card and then install its driver. As each additional sound card is installed, make sure that each driver is installed into a separate directory. This will prevent one driver from overwriting the previous one. It is always suggested that a professional install computer hardware.

DirectX 7.0 or a later version must be installed. DirectX is the advanced application programming interface suite for multimedia functions, designed for Microsoft operating systems. DirectX is not an ordinary program. It cannot be uninstalled from the computer without corrupting Windows. Once DirectX has been installed, this component will need to be installed into Visual Basic by checking the "DirectX7 for Visual Basic Type Library" box located in the References area of the Project menu. Once installed, DirectX is ready to be implemented.
Place the following text in the General_Declarations:

Dim gdx as New DirectX7

Dim gds as DirectSound

Dim m_ds as DirectSound

Dim gdsb as DirectSoundBuffer

Dim mdsb as DirectSoundBuffer

Dim gd as DSBUFFERDESC

Dim $\mathrm{gw}$ as WAVEFORMATEX

Dim dse as DirectSoundEnum

Dim sfile 1

Dim sfile2

$G d s b$ and $m d s b$ are the variables given to the sound buffers. The variable $g w$ gives the waveform information. If the sound buffers are taken directly from sound files, the wave format (i.e., the number of channels and sample rate) will be extracted automatically from each file. The sample rate that is set is the same for each channel. That is, if the sample rate is set at $44100 \mathrm{kHz}$ (CD quality), the sample rate per channel will be $44100 \mathrm{kHz}$, regardless of the number of sound cards (personal communication from Creative Labs, August 13, 2002; Audio Engineering Society, 1992). The variable $d s e$ identifies the total number of sound cards installed in the computer and collects the information concerning each card.

Create two labels on the form and place the following in the Form_Load:
Set dse $=$ gdx.GetDSEnum
dse.Getcount
Label1.Caption $=$ dse.GetDescription $(2)$
Label2.Caption $=$ dse.GetDescription $(3)$
sfile 1 = "c: |windows $\backslash$ medialtada.wav"
sfile2 = "c: $\mid$ windows $\backslash$ medialchord.wav"
loadsoundbufferfromfile1 (sfile1)
loadsoundbufferfromfile2 (sfile2)

Set $\mathrm{gds}=\mathrm{gdx}$.DirectSoundCreate $($ dse.Getguid(3))

The first section enumerates the number of sound cards installed in the computer. The dse.Getcount informs the program how many sound cards are installed in the computer. The dse.Getguid(3) tells the computer to gather information for the particular sound cards and to load their respective drivers. If two sound cards are installed in the machine, a value of three must be entered. The first driver is always the basic Windows sound driver. This driver will not utilize the full capabilities of the sound cards. The value entered should, therefore, always be one more than the number of sound cards installed. The dse.GetDescription variables will display the driver assigned to each sound card value. This is especially convenient, since each sound card is referred to only by its value. By getting the description of each value, it is easy to know which value each sound card is assigned to. This is especially important if one is assigning a particular sound card to play a specific sound buffer. 
The sfile 1 and sfile 2 variables are the sound files to be loaded into the sound buffers. The loadsoundbufferfromfile1 (sfile1) and loadsoundbufferfromfile2 (sfile2) commands load the sound files into their respective buffers.

To create the sound buffers from a file, enter the following:

Private Function loadsoundbufferfromfile1(sfile1 As string) As Integer

If gdsb Is Nothing Then initdirectsound

If sfile $1<>$ vbNullString Then

With gd

.1Flags = DSBCAPS_CTRLVOLUME Or DSB-

CAPS_CTRLPAN

.1 Reserved $=0$

End With

Set gdsb $=$ gds.CreateSoundBufferFromFile(sfile $1_{-}$, $\mathrm{gd}, \mathrm{gw})$

loadsoundbufferfromfile $=0$

Else

loadsoundbufferfromfile $=1$

End If

Exit Function

End Function

Private Function loadsoundbufferfromfile2(sfile2 As String) As Integer

If mdsb Is Nothing Then initdirectsound

If sfile $2<>$ vbNullString Then

With gd

.1 Flags $=$ DSBCAPS_CTRLVOLUME Or

DSBCAPS_CTRLPAN

.1Reserved $=0$

End With

Set mdsb $=$ m_ds.CreateSoundBufferFromFile _

(sfile2, gd, gw)

loadsoundbufferfromfile $=0$

Else

loadsoundbufferfromfile $=1$

End If

Exit Function

End Function

Private Sub initdirectsound( )

Set $\mathrm{m}_{-} \mathrm{ds}=\mathrm{gdx}$.DirectSoundCreate(dse.GetGuid(2)) m_ds.SetCooperativeLevel hWnd _,

DSSCL_NORMAL

Set $\operatorname{gds}=\operatorname{gdx}$.DirectSoundCreate(dse.GetGuid(3)) gds.SetCooperativeLevel hWnd, DSSCL_NORMAL End Sub

Each buffer corresponds to one sound card. Each sound card requires its own sound buffer. The benefit of creating a sound buffer from a file is that the numbers of channels, sample rates, and durations do not need to be specified. All of the information is gathered from each file. The DSBCAPS_CTRLVOLUME and DSBCAPS_CTRLPAN flags allow for volume and panning manipulations. Panning manipulates the sound level of each channel independently.

Each sound card has a corresponding value that needs to be assigned. To assign a particular sound buffer to a specific sound card, change the value of the corresponding dse.GetGuid in the inidirectsound subfunction. The amount of resource sharing can be varied by adjusting the cooperative level. Allocating the default quantity of resources (i.e., very few resources) allows other programs to utilize unused resources. As the amount of allocated resources increases, fewer resources are available for other programs, and therefore, fewer programs can be run at one time. DSSCL_NORMAL enables the program to smoothly multitask among other programs. However, output is restricted to the default 8-bit format. By adjusting the cooperative level to DSSCL_EXCLUSIVE, DSSCL_PRIORITY, or DSSCL_WRITEPRIMARY, the output can be set to 16- or 32-bit formats.

Create a Command button and copy the following to play each sound buffer:

$$
\begin{aligned}
& \text { gdsb.Play DSBPLAY_DEFAULT } \\
& \text { mdsb.Play DSBPLAY_DEFAULT }
\end{aligned}
$$

Create another Command button and copy the following to stop:

$$
\begin{aligned}
& \text { gdsb.Stop } \\
& \text { mdsb.Stop }
\end{aligned}
$$

Sound intensity is modulated by entering a value between 0 and 10,000 , where 10,000 is equal to $100 \%$ of maximum power (i.e., intensity is modulated in step sizes of .01 of absolute amplitude). The intensity of each sound card can be modulated independently. All flags must be entered before playing a particular sound buffer:

gdsb.SetVolume DSBVOLUME_MIN + 5000 mdsb.SetVolume DSBVOLUME_MIN + 3000

The maximum or minimum sound intensity can also be achieved by entering, for maximum intensity,

$$
\begin{aligned}
& \text { gdsb.SetVolume DSBVOLUME_MAX } \\
& \text { mdsb.SetVolume DSBVOLUME_MAX }
\end{aligned}
$$

and for minimum intensity,

$$
\begin{aligned}
& \text { gdsb.SetVolume DSBVOLUME_MIN } \\
& \text { mdsb.SetVolume DSBVOLUME_MIN }
\end{aligned}
$$

This is particularly useful given that the default intensity of each sound card can be different.

The pan function operates in a similar fashion. This makes it possible to present each channel independently. To present only the right channel, enter a value of 10,000 . To present only the left channel, enter a value of $-10,000$ :

gdsb.SetPan (10000)

mdsb.SetPan (-10000) 
At the time this article was written, DirectX 8 had been released. Although Microsoft states that DirectX 8 is no longer optimized for this method, it is fully supported, and it is unlikely that problems will be encountered. The latest version of DirectX can be obtained from the Microsoft Web site (http://www.microsoft.com/windows/ $\operatorname{directx} /$ ).

The method suggested here provides one example for presenting multiple sounds simultaneously. It has been shown that by increasing the number of stimuli presented to participants, selective attention increasingly affects performance (Lavie, 1995; Lavie \& Tsal, 1994). However, auditory studies of this nature have not been carried out in the past, presumably because the technology was not available. These advances in technology will enable researchers to start to investigate human behavior in settings that are more analogous to the outside world (e.g., the cocktail party phenomenon).

\section{REFERENCES}

Audio Engineering Society (1992). AES Recommended practice for digital audio engineering: Serial transmission format for two channel linearly represented digital audio data. New York: Author.

Chan, J., \& SPEnce, C. (2002). Change deafness, an auditory analog of "change blindness." Journal of Cognitive Neuroscience, 14(Suppl.), 18.

Cherry, E. C. (1953). Some experiments on the recognition of speech, with one and with two ears. Journal of the Acoustical Society of America, 25, 975-979.

Cherry, E. C. (1954). Some further experiments upon the recognition of speech, with one and with two ears. Journal of the Acoustical Society of America, 26, 554-559.

Lavie, N. (1995). Perceptual load as a necessary condition for selective attention. Journal of Experimental Psychology: Human Perception \& Performance, 21, 451-468.

Lavie, N., \& Fox, E. (2000). The role of perceptual load in negative priming. Journal of Experimental Psychology: Human Perception \& Performance, 26, 1038-1052.

Lavie, N., \& Tsal, Y. (1994). Perceptual load as a major determinant of the locus of selection in visual attention. Perception \& Psychophysics, 56, 183-197.

MARS, J. D. (2002). Better latent than never [Digital Pro Sound]. Retrieved August 13, 2002 from http://www.digitalprosound.com/ $\mathrm{Htm} /$ Articles/April/Audio_Latency.htm.

Microsoft. (2000). DirectX (Version 7.0a) [Computer programming language]. Redmond, WA: Author.

Nelson, T. W., Bolia, R. S., Ericson, M. A., \& McKinley, R. L. (1998). Monitoring the simultaneous presentation of multiple spatialized speech signals in the free field. Proceedings of the 16th International Congress on Acoustics and the 135th Meeting of the Acoustical Society of America, 4, 2341-2342.

OPENAL API REFERENCE - AL3D. (2000). [On line]. City: Loki Entertainment Software. Retrieved 2002 from www.openal.org.

Spieth, W., Curtis, J. F., \& Webster, J. C. (1954). Responding to one of two simultaneous messages. Journal of the Acoustical Society of America, 26, 391-396.

Steinberg Media Technologies AG (2002). ASIO. Retrieved 2002 from http://www.steinberg.net/developers/ASIO2SDKAbout.phtml.

Wood, N., \& CoWAN, N. (1995a). The cocktail party phenomenon revisited: Attention and memory in the classic selective listening procedure of Cherry (1953). Journal of Experimental Psychology: General, 124, 243-262.

Wood, N., \& CowAN, N. (1995b). The cocktail party phenomenon revisited: How frequent are attention shifts to one's name in an irrelevant auditory channel? Journal of Experimental Psychology: Learning, Memory, \& Cognition, 21, 255-260.

Yost, W. A. (1997). The cocktail party problem: Forty years later. In R. H. Gilkey \& T. R. Anderson (Eds.), Binaural and spatial hearing in real and virtual environments (pp. 329-347). Mahwah, NJ: Erlbaum.

\section{NOTE}

1. Copies of this program can be attained free of charge by sending an e-mail to Jason Chan at jchan@ ted.ie or charles.spence@ psy.ox.ac.uk.

(Manuscript received November 27, 2001; revision accepted for publication August 8, 2002.) 